\title{
PROBLÈMES DES DÉESSES TAŠMĒTUM ET NANAIA
}

\author{
Eiko Matsushima \\ Musée de l'Orient Anient, Tokyo
}

J'ai eu récemment l'occasion d'étudier un certain nombre de textes concernant le Mariage Sacré au Ier millénaire en Babylonie et en Assyrie. Ce Mariage Sacré, assez différent de celui qui est connu dans les documents sumériens, était le plus souvent effectué, d'après ce que nous savons actuellement, par le dieu Nabû et son épouse, tantôt Tašmētum tantôt Nanaia.(1)

Il est bien connu que dans les textes du Ier millénaire, l'épouse de Nabû était appelée parfois Tašmēum, parfois Nanaia. Qu'est ce que cela signifie? Est-ce que cela veut dire que Nabû avait deux épouses distinctes? Ou bien que Tašmētum et Nanaia, étaient tous deux les noms d'une seule divinité?

Ge genre de problème apparaît fréquemment dans l'histoire de la religion assyro-babylonienne. Il est dû à un phénomène de syncrétisme entre plusieurs divinités du panthéon. La question est toujours très compliquée et il est à coup sûr difficile de lui trouver une conclusion. Mais il n'en est pas moins utile d'analyser de temps en temps une telle situation, parce qu'elle peut nous révéler certains points intéressants en ce qui concerne l'histoire de la religion. Dans le cas de Tašmētum et Nanaia, le problème pose précisément une difficulté comme celle qui vient d'être décrite. Nous allons toutefois essayer d'en faire une étude plus ou moins poussée, en analysant les documents qui concernent chacune des deux déesses.

Tašmētum est connue en Babylonie depuis l'époque ancien-babylonienne. Comme son nom (purement sémitique) l'indique, c'est la déesse de l'“entendement" (tašmetu) et de l'“exaucement".(2) Elle était dès cette époque tenue pour l'épouse de Nabû, qui n'était alors qu'un dieu de second rang.

A l'intérieur d'une liste de noms divins de l'époque ancien-babylonienne dite "Liste Weidner", éditée par ce dernier dans AfK 2 (1944),( ${ }^{(3)}$ pp. 1-82, Tašmētum est placée juste après Nabû;

${ }^{d} A M A R . U D \quad{ }^{d} M a r d u k$

Vol. XVI 1980 


$\begin{array}{lll}{\left[{ }^{d} \text { Zar-pa-ni-tum }\right]} & { }^{d}[\text { Zar-pa }]-n i-t u m & \\ { }^{d}[\mathcal{N} a-b i-u m] & { }^{d} \mathcal{N} a ́ b \hat{u}(\mathrm{PA}) & (3 \mathrm{a}) \\ { }^{d}[\mathcal{N} a b \hat{u}] & { }^{d} \mathcal{N} a ́ b \hat{u} & (3 \mathrm{~b}) \\ \left.{ }^{d} \text { Taš-[me-tum }\right] & { }^{d} \text { Taš-me-tum } & \\ & \text { ("Liste Weidner" } & \text { III } 1-4=K A V \text { II } 63 \text { I2-16) }\end{array}$

Dans une autre liste de dieux également ancien-babylonienne, AO 5376, publiée par $\mathrm{H}$. de Genouillac in TCL XV, planches XXV-XXXI, et éditée par le même auteur dans $R A 20$ (1923) pp. 90 et suivantes, Tašmētum est mentionnée juste après Nabû. Ainsi;

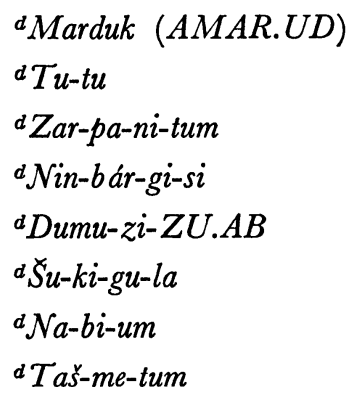

(AO 5376 col. III $=$ TCL pl. XXVI 7-14)

On voit ici que Nabû et Tašmētum appartenaient bien au groupe de dieux de la cour de Marduk. Dans "An=Anum", autre grande liste divine dont l'origine, au moins, monte à cette même époque, Nabû et Tašmētum apparaissent également dans la cour de Marduk (CT 24 pl. 26 II 242-251). Les trois documents cités ci-dessus semblent indiquer que cette déesse dépendait complètement de son époux Nabû.

En dehors des listes divines, il n'y a pas beaucoup de citation concernant Tašmētum. Son nom figure dans l'appellation de la 4le année de Hammurabi: $m u{ }^{d} T a s ̌-m e-t u m ~ i n i m . s a_{6} . s a_{6}$.ga.a.ni "l'année où la parôle de Tašmētum fut favorable".(4)

Cette déesse apparaît isolémemt, à notre connaissance, dans les documents ancien-assyriens. Quant à Nabû, il n'est jamais mentionné dans ceux-ci.(5) A l'époque médio-assyrienne, Tašmētum semble être devenue l'objet d'un respect assez considérable. Elle est invoquée dans une prière de TuklutiNinurta I (1244-1208 avant J.-C.), KAR 128, avec les autres grands dieux et les déesses d'Assyrie (rev. II 31).

Nanaia est connue en Babylonie depuis l'époque ancien-babylonienne. C'est une déesse d'Uruk, fille d'Anu (ou parfois de Sîn), et la patronne de 134

ORIENT 
l'amour. Elle est citée dans la liste divine AO 5376 avec les autres dieux du groupe de Dumuzi;

$$
\begin{aligned}
& { }^{d} \mathcal{N} a-n a-a \\
& { }^{d} Z i l-z i l \\
& { }^{d} \text { In-nin-sa }{ }_{6}-g a \\
& { }^{d} M \text { M }^{\prime} \text { ati }(P A)^{a . t \imath}
\end{aligned}
$$

(TCL XV pl. XXV 34-37)

On la trouve aussi mentionnée dans la "Liste Weidner", également dans le groupe de Dumuzi, mais seule, et Mu'ati n'y figure pas. Ainsi pouvons-nous nous rendre compte que Nanaia, à l'époque ancienne, appartenait à un groupe tout à fait différent de celui où figuraient Tašmētum et Nabû. ${ }^{(6)}$

Il semble que Nanaia était déjà, à l'époque ancien-babylonienne, une grande déesse, à laquelle on a dédié plusieurs hymnes et prières. Nous pouvons citer ici quelques lignes d'un hymne en son honneur, composé au profit de Samsuiluna (vers 1749-1712 avant J.-C.), le septième roi de la Première Dynastie de Babylone;

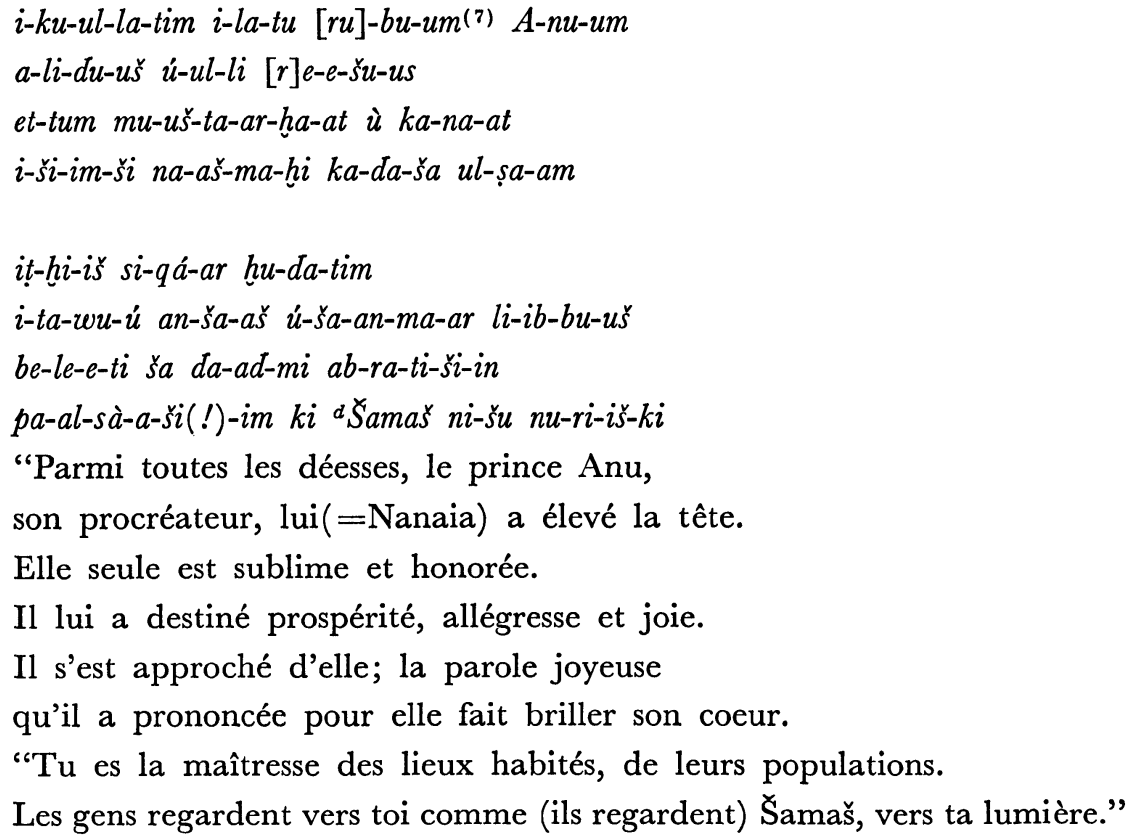

Plus tard, on exalte la bienveillance de la déesse à l'égard du roi Samsuiluna; [da-]ri-a-am ba-la-a-ta-am ${ }^{d} \mathcal{N} a-n a-a^{(8)}$ ar-k[a-am] 
[ta-]tu-ú-ra-am te-și-ip ta-aš-ru-uk-su-[um]

[a-n]a Sa-am-su-i-lu-na na-ra-mi-i-ša

tu-ša-at-[li-]im-šu-um ša-am-ša ki na-an-na-a-ri-im

"Une vie durable et longue, Nanaia

la lui a ramenée, doublée et offerte.

A Samsuiluna, son aimé,

Elle a octroyé le soleil comme lumière céleste."

(ibid., rev. 21-24)

Nous voyons dans les passages cités ci-dessus que Nanaia jouait un rôle très important en tant que protectrice du roi et du pays.

A cette époque, l'époux de Nanaia était Mu'ati, dont nous avons déjà vu le nom dans la liste divine AO 5376. Il y a un texte lyrique datant du règne d'Abi-ešuh, successeur de Samsuiluna (vers 1711-1684 avant J.-C.), VAT $13747,{ }^{(9)}$ qui nous donne des renseignements intéressants à propos de ce couple divin. On y trouve Nanaia et Mu'ati se parlant entre eux; et encore un troisième personnage qui joue le rôle de récitant. A la "face" de la tablette,(10) Mu'ati invite Nanaia à faire l'amour avec lui; après plusieurs lignes, vers la fin, le récitant demande à Nanaia de bénir le roi Abi-ešuh et de lui assurer une vie longue. Au "revers", on exalte la déesse en tant que protectrice de la ville de Babylone, puis on parle de l'amour du couple divin. Ce texte fait probablement allusion à une cérémonie du Mariage Sacré de Nanaia et Mu'ati, au profit du roi et de la ville de Babylone.

$\mathrm{Au}$ point de vue de l'histoire de la déesse Nanaia, il y a deux choses à noter. D'abord, on y décrit une hiérogamie de l'époque ancien-babylonienne, dans laquelle transparaît encore la tradition du Mariage Sacré, souvent mentionnée dans les documents sumériens. ${ }^{(11)}$ Nanaia est, bien entendu, la déesse de l'amour, comme une autre grande déesse d'Uruk: Ištar. C'est sans doute pour cela qu'on la trouve dans un texte comme VAT 13747. Mais dans ce dernier, en plus, elle est priée d'assurer longue vie au roi et, à la requête qui lui est faite, elle considère Babylone avec des yeux favorables, la bénit, en décrète la prospérité et assure au roi une bonne santé. Voici un passage du texte en question;

$$
\begin{aligned}
& \text { ta-ap-pa-l[i-is Bä]bilam } \left.(K \bar{A} . D I N G I R)^{k t} \text { i-na i-ni-ša dam-[qa-tim } \ldots . . . .\right] \\
& t a-a k-r u-u b[-s u t] a-a q-t a-b i \quad d u-m u-u q-s u[\ldots . . .]
\end{aligned}
$$

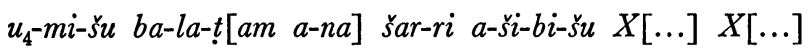

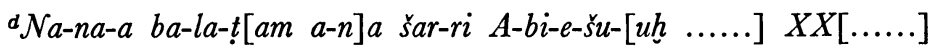

$$
\begin{aligned}
& t u-s ̌ u-s ̌ i-i b-s ̌ u ~ i-n a ~ s ̌ u-p a-a t ~ n i-i h-t i l i-X[\ldots . .] \text { im-ma }[\ldots . . .]
\end{aligned}
$$


"Elle regarde Babylone de ses yeux favorables.

Elle la bénit. Elle ordonne sa prospérité.

Chaque jour ...... la vie pour le roi Abi-ešuh ......

Elle l'installe en une résidence calme."

(VAT $13747 \operatorname{rev}(?)$ 3-7)

Nous pouvons ainsi comprendre que Nanaia a acquis certaine importance et est devenue l'objet d'un respect assez considérable de la part des rois de la Première Dynastie de Babylone. Comme nous l'avons déjà vu dans les listes des dieux, Nanaia n'avait rien à voir, à cette époque, ni avec Nabû, ni avec Tašmētum. Nabû n'était d'ailleurs qu'un dieu de second rang dans le panthéon, et nous n'avons pas beaucoup de citations le concernant.(12)

Or, environ un demi millénaire plus tard, dans un kudurru de MérodachBaladan I (1173-1161 avant J.-C.), il y a un passage où l'on lit;

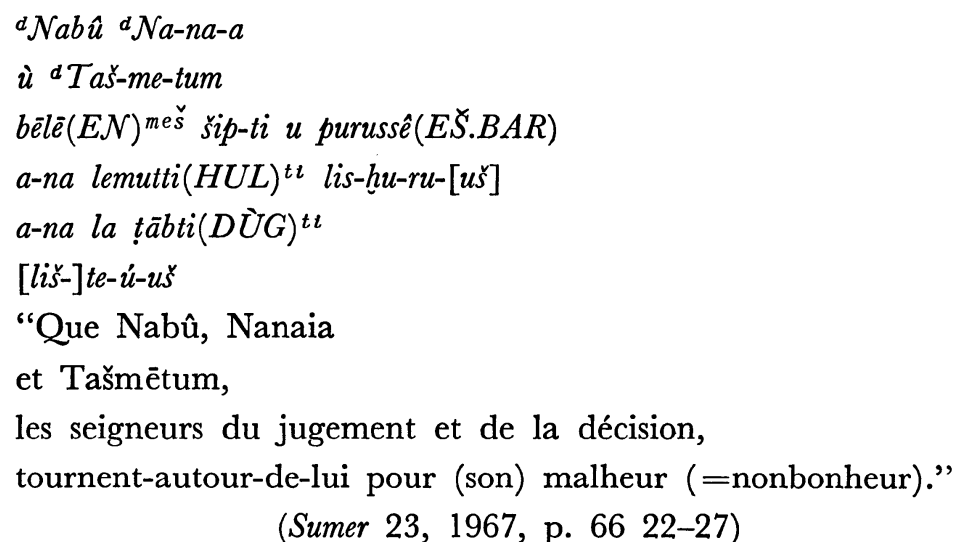

C'est en fait le document le plus ancien connu aujourd'hui, où l'on trouve Nabû et Nanaia placés côte à côte. On ne sait pas comment interpréter exactement les relations entre les trois divinités énumérées dans ce passage; mais il est intéressant que Tašmētum ait été mentionnée après Nanaia. Il semble que l'on distinguait alors les deux déesses, Nanaia et Tašmētum, en les regroupant toutefois autour de Nabû. G'est là cepandant, à notre connaissance, l'exemple unique au IIe millénaire, où figurent ensemble Nanaia et Nabû. On les trouve quelquefois dans des kudurru, mais séparément. En général, Nanaia paraît appartenir au groupe des dieux d'Uruk, et Nabû à celui de Marduk, et donc de Babylone. ${ }^{(13)}$

Dans un texte d'incipits de chants, en moyen-assyrien, $K A R$ 158,(14) Nanaia figure à plusieurs reprises; elle y est décrite en tant que protectrice du pays et 
déesse de l'amour, comme Ištar. Par exemple;

${ }^{d} \mathcal{N a}$-na-a ma-ti-ma

šul-ma a-na šarri(LUGAL) ki-i ta-ši-mi

tuk-te-en-ni šar-ra-tu ${ }^{d} \mathcal{N a} a-n a-a$

am-ma-rat kal niš̂̀ $(U \mathcal{N})^{m e s ̌ s}$ ra-'-um-ta

"(C'est) toujours, ô Nanaia,

que tu destines la sécurité au roi.

Tu es bien placée, la Reine Nanaia.

Celle qui regarde tout les peuples aimés."

(KAR 158 II 23-26)

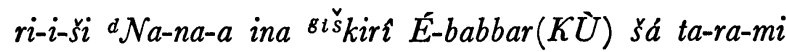

"Réjouis-toi, ô Nanaia, dans le jardin de l'Ebabbar que tu aimes".(15)

(ibid., VII 38)

A la colonne $\mathrm{V}$ de $K A R$ 158, qui est si fortement cassée qu'on n'y lit que difficilement quelques lignes, il y a un passage qui nous fait penser à Tašmētum (et aussi à Nabû). Par exemple, l'expression comme i-la-at ta-áš-me-e ... "la déesse de l'entendement" (V 13) est une épithète fréquente, et presque exclusivement employée à propos de Tašmētum. Nous pouvons ainsi savoir que Nanaia a gardé son propre caractère et son rôle principal, qui est celui de patronne de l'amour et de protectrice du pays, dans ce document qui date de l'époque moyen-assyrienne.

$\mathrm{Au}$ premier millénaire, nous avons des textes où Nabû et Nanaia sont décrits comme époux-épouse. Dans un acte de donation de Marduk-zākir-šumi (854-859 avant J.-C.), (16) on lit;

${ }^{d} \mathcal{N} a b \hat{u} u^{d} \mathcal{N} a-n a-a$

ilāni(DINGIR.DINGIR) șirūtit $(M A H)^{m e s ̌ ~}$

$i s[i d(S U H U S)-s] u \quad l i-i s-s u-h u$

$\grave{u}[z \bar{e} r-] \check{s} u$ lil-qu-tu

"Que Nabû et Nanaia,

les dieux sublimes,

arrachent sa base, ${ }^{(17)}$

et suppriment sa prospérité!"

(RA 16, 1919 p. 126 IV 2-5)

Le couple divin se place, dans ce texte, juste après Marduk et Zarpānîtum (III 31 - IV 1), et on voit bien clairement que Nanaia, en tant qu'épouse de 
Nabû, est censée appartenir au groupe des dieux de la cour de Marduk.

Dans le kudurru de Nabû-šum-iškun (760-748 avant J.-C.), il y a;

${ }^{d} \mathcal{N a}$-na-a beltu sir-ti

šur-bi-ti ilānimeš $q a-r i t-t i$

$i$-lat $i$-la-a-ti ša-qut bēlètimešs

se-ma-ti su-pe-e

ma-hi-rat un-nin-nu hi-rat ${ }^{d} \mathcal{N} a b \hat{u}$

ta-lim-mat d Samšisì na-ram-ti ${ }^{d}$ Marduk

bu-kur-ti ${ }^{d} A-n u$ res-ti-ti

sa ki-bit-su ma-ag-rat

ša ina puhur ilāni ${ }^{m e s ̌}$ rabütimešs

la iš-ša-an-na-nu be-lut-su

ma-li-kat d Igigi mu-šad-di-ḩat har-ra-nu

ša ina É-sag-íl ekal šamê u erșêti

šit-lu-ța-at be-lut-su

"Nanaia, la Dame sublime,

La plus grande des dieux, la vaillante,

La déesse des déesses, la plus haute des souveraines,

Celle qui écoute les prières,

Celle qui reçoit l'invocation, l'épouse de Nabû,

La soeur de Samaš, l'aimée de Marduk,

La fille aînée première en rang d'Anu,

(Lui) dont la parole est favorable,

dont, dans l'assemblée des grands dieux,

la souveraineté est sans rivale.

La princesse des Igigi, qui avance solennellement sur la voie, (celle) de qui dans l'Esagil, le temple des cieux et de la terre, la souveraineté domine."

$$
\text { (VS I } 36 \text { I 1-13=RA } 16 \text { 141-144)(18) }
$$

Ici, Nanaia est à la fois l'épouse de Nabû (I 5) et la fille d'Anu (I 6). Elle est décrite d'une part comme une très grande déesse qui a beaucoup de pouvoir (1-3, 7 et suivantes) - trait qu'elle gardait depuis le IIe millénaire; mais, d'autre part, elle est "l'aimée de Marduk" et "la reine de l'Esagil". Aussi peut-on noter les expression comme "elle écoute les prières" et "elle reçoit l'invocation". Ce sont les phrases utilisées normalement pour exalter Tašmētum. (19) Faut-il voir là une sorte de confusion entre Nanaia et celle-ci? Il Vol. XVI 1980 
est en tout cas certain qu'aux IXe et VIIIe siécles, Nanaia en était venue à être considérée comme l'épouse (légitime) de Nabû, tout en gardant son propre caractère.

A l'époque récente, c'est-à-dire aux périodes néo-assyrienne et néo-babylonienne, on trouve un bon nombre de citations relatives à Tašmētum et Nanaia. Tašmētum, étant l'épouse de Nabû qui était devenu, au Ier millénaire, un des dieux les plus importants du panthèon, a acquis alors une position assez considérable. Nous pouvons citer ici quelques passages d'hymnes dédiés à cette déesse: par exemple;

[mu] ${ }^{\xi}$-te-eš-ma-at a-mat-sa šá-ki-na-at taš-me-e [mu]š-te-ni-ih $u z-z i$ ili $u$ iš [täri]

se-mat tes-li-ti u su-pe-e

li-qat ik-ri-bi u un-nu-ni

hi-rat(!) sar-hi ${ }^{d} M u{ }^{\prime} a t i(P A)^{a . t t}$ a-pil ${ }^{d} T u$-tu

kal-lat(!) É-sag-il du-ru-uš šamê(?) šadûu kib-ra-a-ti

$z \bar{e} r(\mathcal{N} U M U \mathcal{N})$ É-zi-da bit ši-kin na-piš-ti ilänimešs rabūtimeš

sar-rat Bár-sipak ba-'-lat da-ád-me

${ }^{d}$ Taš-me-tum be-el-tum šá qí-bi-sa gaš-rat

"Celle qui fait écouter sa parole, celle qui accorde l'entendement.

Celle qui calme la colère du dieu et de la déesse.

Celle qui entend prière et supplication.

Celle qui reçoit l'oraison et l'invocation.

L'épouse du glorieux Mu'ati, le fils de Tutu.

La belle fille de l'Esagel, le base des cieux, la montagne des quatres coins du monde.

La semence de l'Ezida, temple des êtres vivants des grands dieux.

La reine des Borsippa, celle qui gouverne les lieux habités.

Tašmētum, la souveraine dont la parole est très grande."

(L. W. King, BMS 33 f. 2-10)

Le passage exprime bien le caractère essentiel de Tašmētum. G'est une déesse qui exauce, et qui se fait écouter et obéir: elle est intelligente et miséricordieuse. On l'a décrite aussi comme;

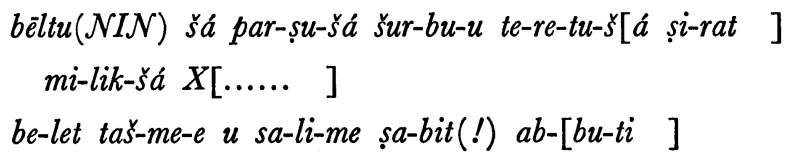


$m u-b a-l i-t a-a t \quad X[\ldots . .$.

"La souveraine, dont la fonction est très grande, dont les charge sont sublimes, dont le conseil est......

La maîtresse de l'entendement et de la paix, celle qui intercède, celle qui fait vivre......"

(KAR 362 f. 3-6)

Tašmētum était aussi patronne des scribes, son épous étant le dieu de l'art du scribe. ${ }^{(20)}$ Enfin, nous pouvons caractériser cette déesse par l'intelligence, la discrétion, la douceur, etc.

Quant à Nanaia, on la trouve mentionée dans les documents de la période de la Dynastie Sargonide assyrienne. Dans une prière de Sargon II (721-705 avant J.-G.), il y a;

im-na u šu-me-la su-ud-dur tam-ha-ru

$a-s ̌ a-r e t-t i$ ilānimešs šá me-lul-šá qab-lum

a-li-kat mah-ri šu-ut si-bit at-he-e

"A (sa) droite et à (sa) gauche sont rangées les forces de la guerre, (Elle) est la première des dieux, et son jeu est le combat,

Elle marche devant ceux qui sont les Sept Frères."

(J. A. Craig, $A B R T$ I $54-55=B A 5 / \mathrm{V}$ p. 626 no. IV, col. I 6-8)

On a impression, ici, qu'elle partage certaines prérogatives d'Ištar qui était non seulement la déesse de l'amour comme Nanaia, mais aussi la déesse de la guerre. Plus tard dans le même texte, il y a toutefois;

kal-lat É-sag-il[ ] $X E X[\quad]$

hi-rat ${ }^{d} M u$ 'ati na-ram-ti ${ }^{a} B \bar{e} l$ [ ]

"La belle-fille de l'Esagil,

l'épouse de Mu'ati, l'aimée de Bēl......

(ibid., II 2-3)

Ce passage nous montre, bien entendu, que Nanaia est censée être l'épouse de Nabû, qui s'était identifié déjà à Mu'ati; ce dernier était, comme nous l'avons vu plus haut, l'époux de Nanaia dès le IIe millénaire.(21)

Il semble que Nanaia avait pris une place importante à certaine époque de la Dynastie Sargonide. Asarhaddon (680-669 avant J.-C.) récitait des éloges de cette déesse. (22) Assurbanipal (669-630 avant J.-C.) explique, dans une de ses inscriptions royales, qu'à l'occasion de son éxpédition au pays d'Elam, Vol. XVI 1980 
il a ramené cette déesse qui s'en était allée de Babylonie il y a longtemps; le roi a pris, lui même, la main de Nanaia, pour l'introduire dans le sanctuaire qu'Assurbanipal avait renouvelé pour elle à Uruk. ${ }^{(23)}$

Nous avons ailleurs un hymne bilingue, composé à l'époque, dans lequel Nanaia s'exalte elle même, en s'identifiant à plusieurs grandes déesses de Babylonie et d'Assyrie. ${ }^{(24)}$ Dans les textes incantatoires du genre $\zeta \grave{A} . Z I . G A$, elle figure souvent en tant que déesse de l'amour (-sexuel), comme Ištar qui se présente, elle aussi, souvent dans ce genre de textes.(25)

On se rend compte ainsi que Nanaia, au moins dans les textes cités cidessus, paraît avoir un caractère très différent de celui de Tašmētum. Nanaia est tout d'abord la patronne de l'amour; elle est parfois même puissante et vaillante. Mais nous avons vu qu'elle est aussi appelée "l'épouse de Nabû". Comment peut-on comprendre ces faits?

Parmi les formules de salutation figurant dans les lettres néo-assyriennes, nous en avons quelques unes où se trouvent Tašmētum et Nanaia ensemble: par exemple;

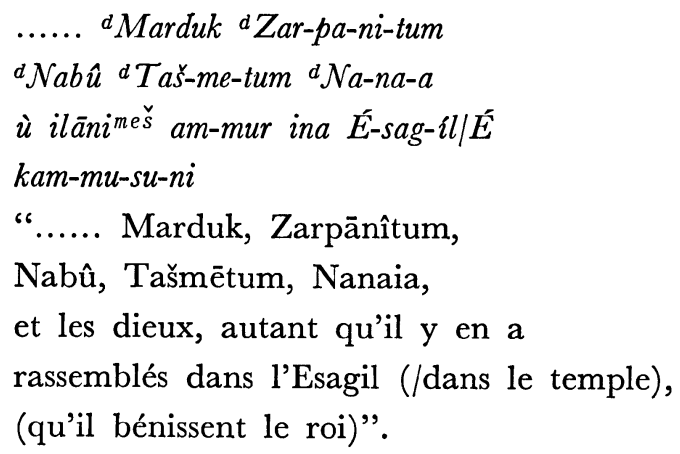

( $A B L 119$ f. 5-8, $A B L 120$ f. 5-8)

Et encore dans une autre lettre;

.....UD.16.KÁM

UD.17.KÁM ša pān ${ }^{m u l}$ Dil-bat ${ }^{d} \mathcal{N} i n-l i ́ l$

${ }^{d}$ Zar-pa-ni-tum ${ }^{d}$ Taś-me-tum ${ }^{d} \mathrm{Gu}$-la

${ }^{d} \mathcal{N} a-n a-a$ is-se-niš ep-pa-ás

"Le seizième jour

et le dix-septième jour, j'exécuterai tous (ces rites) ensemble devant la (déesse de la) Planète-Vénus, Ninlil,

Zarpānîtum, Tašmētum, Gula

et Nanaia." 
(ABL 23 rev. 2-5)

Tout ces exemples semblent nous démontrer qu'on a distingué alors bien nettement Tašmētum de Nanaia. Il est intéressant d'observer que, dans les lettres néo-assyriennes citées ci-dessus, Tašmētum est mentionnée avant Nanaia, tandis que, dans le kudurru de Mérodach-Baladan (cité plus haut) la dernière se plaçait avant la première.

Nous savons bien qu'àux époques néo-assyrienne et néo-babylonienne, la cérémonie du Mariage Sacré de Nabû et de son épouse, tantôt Nanaia, était effectuée. D'après les lettres néo-assyriennes, nous pouvons reconstituer, en gros, ce rite: au mois d'Ajjar (avril - mai), à Kalaḩ (et peut-être aussi, dans les autres grandes villes d'Assyrie), on célébrait le rite de la Hiérogamie de Nabû et Tašmētum. Le couple divin entrait dans "la chambre à coucher", c'est-à-dire le sanctuaire préparé pour l'occasion, et y restait pendant un certain temps. On faisait un sacrifice devant les dieux dans l'espoir qu'ils assurent "une longue vie" aux fils du roi et la prospérité à la famille royale.(26) Il semble que la cérémonie était accompagnée de musique. Nous connaissons un texte du style "chant d'amour" relatif à Nabû et Tašmētum, en néo-assyrien, qui a dû être utilisé à cette occasion.(27)

Le rite avait lieu aussi en Babylonie. Là, la Hiérogamie était effectuée entre Nabû et Nanaia. Selon un calendrier cultuel $S B H$ no. VIII, le rite avait lieu au mois d'Ajjar, sans doute à la ville de Borsippa, mais dans le temple de Nanaia et le jardin d'Anu, père de la déesse. Nabû prenait la couronne d'Anu au cours de la cérémonie, ce qui nous amène à penser que cette couronne possédait certaine signification symbolique. ${ }^{(28)}$

Nous n'examinerons pas maintemant en détail le problème de cette Hiérogamie. Mais il est au moins important de noter que les documents assyriens citent Tašmētum comme l'épouse de Nabû dans la cérémonie en question, tandis que les documents babyloniens font mention de Nanaia. Il est vrai qu'en Assyrie, Tašmētum avait une position assez importante depuis le IIe millénaire, comme nous l'avons vu plus haut. Il y a - au moins d'après nos connaissances actuelles - davantage de citations relatives à Nanaia en Babylonie qu'en Assyrie, même si cette déesse a été l'objet d'un respect considérable de la part des rois assyriens de la Dynastie Sargonide. Plus tard, les souverains de la Dynastie Chaldéenne notaient plutôt le nom de Nanaia que celui de Tašmētum dans leurs inscriptions royales. ${ }^{29)}$ Il semblerait donc que Tašmētum était l'épouse "assyrienne" de Nabû et que Nanaia était celle de "Babylonie". 
Pourtant, ce n'est là qu'une hypothèse dont nous n'avons aucune preuve définitive: Tašmētum paraît être à l'origine une déesse babylonienne (sémitique), ${ }^{(30)}$ tandis que Nanaia est décrite quelquefois, même dans les documents neo-assyriens, comme l'épouse de Nabû.

Ainsi avons nous examiné les documents dans lesquels apparaissent les caractères et les situation de chacune des deux déesses. Au IIe millénaire, c'étaient deux divinités totalement distinctes. Et il nous paraît que chacune gardait encore au Ier millénaire son originalité.

Mais, dans le kudurru de Nabû-šum-iškun, nous avons vu déjà que, parmi les expressions utilisées pour exalter Nanaia, il y a šemäti supē//mahirat unnini "celle qui écoute les prières, celle qui reçoit l'invocation"; ce sont des épithètes employées plus souvent pour Tašmètum. Il serait en conséquence possible de supposer qu'on a confondu alors dans une certaine mesure, Nanaia avec Tašmētum.

Il y a encore un petit nombre de documents que nous pouvons examiner. Dans une incantation du genre $S \grave{A} . Z I . G A$, on trouve;

${ }^{d}$ Taš-me-tum kal-lat ${ }^{d}$ Marduk pa-šu-mu iš-kun-[k]i

${ }^{d} \operatorname{Sin}(X X X)$ bèl mi-lam-me-e ana libbi $(\zeta \grave{A})$-ki iš-kun $X X$

$m \bar{a} r(D U M U){ }^{d} A-n i m$ qar-du i-nam-di-[in]

bu-un-na-ni-ki tê šipti( $\left.T U_{6} . E \mathcal{N}\right)$

"Tašmētum, la belle-fille de Marduk, t'a mis un voile.

Sîn, le seigneur de la splendeur, a mis......à ton coeur.

Le vaillant fils d'Anu a établi

ta figure. Formule de l'incantation."

(KAR 69 f. 20-rev. 1=R. Biggs, $\zeta \grave{A} . Z I . G A$ pp. 75-76, dans Appendix)

Il s'agit là d'une sorte d'incantation d'amour (-sexuel); Tašmētum y est décrite, d'une part comme "belle-fille de Marduk", et d'autre part, comme une déesse qui appartient au groupe des dieux d'Uruk (Anu, Sîn, Ištar etc.). Nous pourrions donc peut-être voir ici une sorte de confusion de Tašmētum avec Nanaia.

A l'époque néo-babylonienne (Nabopolasar et Nabuchodonosor), on a unnom

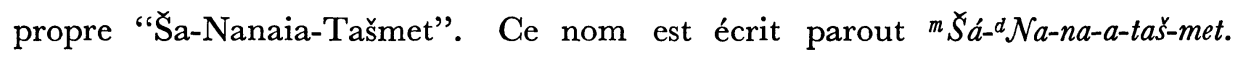

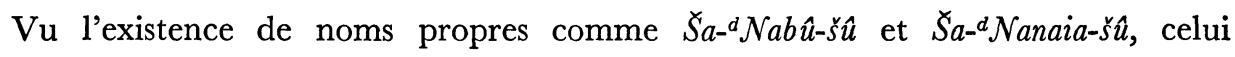
qui nous intéresse est certainement du même type, avec supression du pronom 
final, et ne peut signifier "(Qui appartient) à Nanaia-Tašmet". l'interprétation la plus vraisemblable qu'on puisse en donner est de faire de Tašmet une apposition de Nanaia; les deux appellations sont donc équivalentes, Tašmet étant considéré comme une sorte d'épithète, ou de "second nom" de Nanaia. Il est plus difficile, étant donné la graphie absoule tašmet, d'entendre "De Nanaia (provient) l'exaucement". En conclusion, nous pouvons déceler ici une tendence consciente à identifier Nanaia et Tašmētum.

Nous avons ainsi étudié le problème de Tašmetum et Nanaia, deux déesses qui étaient considérées comme les épouses de Nabû au Ier millénaire. Il semble qu'on puisse admettre les choses suivantes:

1) Jusqu'à la fin du IIe millénaire, Tašmêtum et Nanaia étaient deux divinités totalement distinctes. Tašmêtum était l'épouse de Nabû en Babylonie et tous deux appartenaient à la cour de Marduk. Il apparaît que Tašmêtum avait acquis une position assez importante en Assyrie. Nanaia, grande déesse d'Uruk, avait son propre époux, Mu'ati, et jouait toujours un rôle important en tant que patronne de l'amour et ptotectrice du pays.

2) Vers la fin du IIe millénaire, on a commencé à rapprocher Nabû de Nanaia.

3) Au Ier millénaire, Tašmêtum et Nanaia étaient appelées l'une et l'autre "I'épouse de Nabû". En général, chacune avait son propre caractère et son propre rôle. Il semble donc qu'on ait distingué assez nettement ces deux divinités. Il y a plus de références concernant Tašmêtum en Assyrie, et d'avantage à propos de Nanaia en Babylonie. Mais il faut relever que cette dernière figure souvent dans les documents assyriens de la Dynastie Sargonide.

4) Il existe un petit nombre de documents, surtout d'époque tardive, où nous trouvons une sorte de confusion volontaire ou une tendance à identifier consiemment Tašmêtum et Nanaia.

Disons, pour terminer, qu'il est difficile, et presque impossible de tirer des conclusion sûres relativement à ce problème. Nous savons que dès la fin de IIe millénaire, Nabû avait été l'objet d'une promotion très rapide dans le panthèon. C'est justement à cette époque que nous avons le document - le kudurru de Mérodach-Baladan I - dans lequel Nanaia est mentionée avec Nabû (et aussi avec Tašmêtum). Après peu de temps, Nanaia était devenue Vol. XVI 1980 
l'épouse de Nabû. Nous sommes alors tentés de supposer qu'on a lié Nabû et Nanaia vers cette époque pour quelque raison particulière. Nous pouvons naturellement penser à la graphie ${ }^{d} M u^{\prime} a t i={ }^{d} P A^{a . t \imath}$ et ${ }^{d} P A={ }^{d} \mathcal{N} a b \hat{u}$, et présumer qu'on a associé Nabû et Nanaia à cause de l'identification préalable de Nabû à Mu'ati. Mais pourquoi à la fin de IIe millénaire? D'ailleurs, la graphie ${ }^{d} P A$ pour Nabû n'est pas attesté de façon assurée avant le Ier millénaire. ${ }^{(32)}$

Or Nanaia était déjà, au IIe millénaire, une déesse fort importante; elle était considérée comme fille d'Anu (ou de Sîn). Est-il déraisonnable de penser que Nabû, qui était devenu de plus en plus important dans le panthéon, était alors digne d'avoir une grande déesse comme Nanaia pour son épouse? Plus tard, il allait jouer quelquefois le rôle de "gendre" d'Anu, le chef des dieux du panthéon antique, comme on le voit dans le texte $S B H$ no. VIII.

Certes, il s'agit là d'une hypothèse touchant laquelle nous n'avons pas, pour l'instant, assez de matériaux pour juger si elle est acceptable ou non. De toute manière, Nanaia est censée être alors, d'ores et déjà, l'épouse de Nabû. Pourtant ce dieu avait sa propre épouse dequis le IIe millénaire: Tašmētum, qui allait le rester au cours du Ier.

En général, on a continué, semble-t-il, à appeler chacune des deux déesses "I'épouse de Nabû", en distinguant toutefois l'une de l'autre. Mais ce n'était pas là une situation normale et il y avait probablement des gens qui confondaient les deux, ou qui essayaient de voir Tašmētum et Nanaia comme une seule divinité. A notre connaissance, il n'y a, pour l'instant, qu'un petit nombre de documents pour attester ce dernier fait et la tendance d'identifier Tašmētum à Nanaia ou Nanaia à Tašmētum ne semble pas avoir été très répandue.

On trouve fréquement dans l'histoire du panthéon assyro-babylonien, ce genre de syncrétisme entre plusieurs personalités divines, assumée l'une par l'autre, ou plusieurs par une seule, qui prend les traits caractéristiques, les pouvoirs, la personnalité et le nom des plusieurs. Ce peut avoir été le cas de Tašmētum et Nanaia au Ier millénaire. De toute façon, si les rapports précis des deux déesses demeurent obscurs, du moins est-il sûr qu'elles étaient tenues, l'une aussi bien que l'autre, pour les épouses de Nabû et que toutes deux étaient des déesses très importantes. 
* Les abréviations bibliographiques utilisées dans cet article suivent le code établi par R. Borger dans Handbuch der Keilschriftliteralur, II (Berlin 1975), pp. XI et suivantes.

\section{Notes}

(1) La transcription "Nanaia", que nous adoptons dans cet article, semble préférable à "Nanâ", que l'on utilise souvent. Voir R. Biggs, ŠA.ZI.GA, Ancient Mesopotamian Potency Incantaions, TCS II (Locust Valley, 1967) p. 20, commentary 24. (Biggs adopte la transcription Nanaya).

(2) Voir $A H w$ 1339a: Tašmètu(m) et 1352: tašmû́; von Soden traduit "Erhörung".

(3) G'est une liste originaire de l'époque de la IIIe Dynastie d'Ur. La liste, telle qu'elle nous est connue aujourd'hui, se compose de plusieurs fragments: VAT 6563 (copié par E. Weidner dans AfK 2 p. 6), VAT 7759 (également dans $A f K$ 2, pp. 4-5) et St Langdon, W-B 9: OECT I pl. 22 sont les tablettes ancien-babyloniennes. $K A V 46,47,62,63$ et 65 sont les copies tardives trouvées à Assur. Pour les détails, $A f K 2$ pp. 1-9 et $R L A 3$ p. 474 .

(4) Voir RLA 2 p. 181.

( 5 ) Voir H. Hirsch, Untersuchungen zur altassyrischen Religion, AfO Beiheft 13/14, Graz 1961, p. 26.

(6) Nous ne trouvons ni Nanaia ni Mu'ati dans la liste "An=Anum"; mais la partie qui doit contenir le groupe de Dumuzi est gravement endommagée et il est vraisemblable que les noms qui nous intéressent sont perdus à cause de la cassure.

( 7) Von Soden lit [la]-bu-um A-nu-um, et traduit "...le Lion Anu": ZA 44 pp. 32-33. Il maintient dans $A H w 526$, ainsi que dans $S A H G$ p. 238, cette lecture. Nous lisons pourtant [ru]-bu-um, en accord avec $C A D \mathrm{~A} / 1340 \mathrm{~b}$, et $C A D \mathrm{E} 126 \mathrm{~b}$, cette lecture nous semblant plus simple et plus claire. Dans la copie de Zimmern dans VS X, le signe ru/la n'est pas clair parce qu'il est cassé. (8) Dans la copie de Zimmern, la lecture ${ }^{d} \mathcal{N} a-n a-a$ est tout à fait claire. Cependant von Soden ne le traduit pas dans son article de $Z A 44$.

(9) Le texte est publié, avec copie, transcription et traduction, par W. G. Lambert dans "Divine Love Lyrics from the Reign of Abi-ešub", MIO 12 (1966), pp. 41-51.

(10) La tablette est assez gravement endommagée et sa forme n'indique pas clairement quel côté est la face et quel côté le revers. W. G. Lambert lui-même nous prévient que l'ordre qu'il a adopté est provisoire: voir $M I O 12$ p. 41.

(11) Pour la définition de la Hiérogamie, surtout sumerienne, et les discussions la concernent, voir J. Renger, "Heilige Hochzeit; A. Philologisch", RLA 4 pp. 251-259. La bibliographie est assez complète; on s'y reportera pour le sujet en question.

(12) Voir Fr. Pomponio, Nabû, il culto e la figura de un dio del Pantheon babilonese ed assiro, Studi Semitici 51 Roma 1978, pp. 15-45.

(13) Voir par exemple, L. W. King, BBSt no. 6 col. II 48, A. Goetze, JCS 19 (1965), UIOM 2499 rev. 31, St. Langdon, OECT 1, pls. 20-21.

(14) C'est un long texte trouvé à Assur et publié par E. Ebeling dans $K A R$, sous le numéro 158. L'auteur a présenté une édition du texte en question dans Berliner Beiträge zur Keilschriftforschung, 1/III (1922), pp. 1-32, "Ein Hymnenkatalog aus Assur". Mais son travail est malheureusement insuffisant à plusieurs points de vue, et une nouvelle édition serait nécessaire. Il s'agit là d'une liste de chants lyriques en sumérien et en akkadien. Aux colonnes VI et VII, nous trouvons des incipits de chants dont les sujets tournent autour de thèmes amoureux, et Nanaia y apparaît souvent (outre les exemples cités ici, il existe un certain nombre de chants qu'on peut supposer dédiés à Nanaia).

(15) Il est intéressant de noter que l'on mentionne ici le jardin de l'Ebabbar (de Larsa), où la déesse se réjouit, alors que Nanaia est à l'origine, la déesse de la ville d'Uruk, et non pas celle de Larsa.

Vol. XVI 1980 
(16) Voir Fr. Thureau-Dangin, “Un acte de donation de Marduk-zâkir-šumi”, $R A 16$ (1919), p. 117 et suivantes.

(17) C'est-à-dire "la base" de celui qui veut détruire ce kudurru.

(18) L' édition du texte est présentée par Fr. Thureau-Dangin, dans $R A 16$ pp. 141-144, dans l'appendice de l'article cité ci-dessus.

(19) Voir K. Tallqvist, $A G E$ p. 471.

(20) H. Hunger, Babylonische und assyrische Kolophone, AOAT 2, Neukirchen-Vluyn, 1968, passim. (21) Le problème de Nabû et Mu'ati est discuté par W. G. Lambert dans $M I O$ 12, pp. 43-48, et également par Fr. Pomponio dans op. cit. (note 12), pp. 40-42.

(22) Voir R. Borger, Die Inschriften Asarhaddons, König von Assyrien, AfO Beiheft 9, Graz 1956, $\S \S 49-50$.

(23) Voir M. Streck, Assurbanipal, VAB 7/II, Leipzig 1916, p. 174.

(24) E. Reiner, "A Sumero-akkadian Hymn of Nanâ", JNES 33 (1974), pp. 221-236.

(25) Voir R. Biggs, Ц̌́̀. ZI.GA, TCS II.

(26) Nous pouvons reconstituer la cérémonie en Assyrie à l'aide des $A B L 65,113,336 . A B L 65$ parle de celle exécutée à Kalah (f. 7), mais ce qui est des $A B L 113$ et 336, nous sommes incapables d'identifier la ville où la cérémonie avait lieu. Cependant, les détails du rite décrit dans ces trois lettres se ressemblent bien et mous présumons donc qu'il se rapportent à la cérémonie du Mariage Sacré de Nabû et Tašmetum, réalisée au mois d'Ajjar dans certaine(s) grande(s) ville(s) en Assyrie. Les lettres datent toutes les trois du règne d'Asarhaddon. Sur ce sujet, J. N. Postgate a écrit un article "The Bit-Akîti in Assyrian Nabû Temples" dans Sumer 30 (1974), pp. 51-72. Moi-même, j'ai fait à ce sujet des recherches dans la thèse que j'ai présentée en octobre 1980 à l'Université de Paris I.

(27) J. van Dijk, TIM IX (1976), no. 54 (publié antérieurement par le même auteur dans Sumer 13, 1957, planches XXVI-XXVII). La transcription et la traduction du texte se trouvent dans la thèse cité à la note précédente.

(28) Nabû y joue le rôle de "gendre" d'Anu. Vu le contexte, on dirait même que Nabû assume plus ou moins toutes les prérogatives d'Anu, dieu qui avait été chef du panthéon antique, en profitant de son Mariage avec la fille de ce dernier.

(29) Voir St. Langdon, Die neubabylonischen Königsinschriften, VAB 4, Leipzig 1912.

(30) Noter que son nom est mentionné, comme nous l'avons remarqué, dans la formule de la 41e année de Hammurabi, roi de la Première Dynastie de Babylone, ainsi que les citations dans les listes divines que nous avons vues.

(31) Voir H. F. Lutz, Néo-Babylonian Administrative Documents from Erech, UCP 9/I, Berkeley 1927, 26 6, 39 5, 10 57: 10 et R. P. Dougherty, Goucher College Cuneifomr Inscriptions, Archives from Erech I, New Havne 1923, 96 7, 1166 et Ibid., II, New Haven 1933, 3473.

(31) Voir Pomponio, op. cit., pp. 5-13. 